\title{
A sigmoidovesical fistula due to colonic tuberculosis
}

\author{
Matheesan $\mathrm{T}^{1}$, Thoufeek $\mathrm{S}^{2}$, Mahindawansa $\mathbf{I}^{3}$, Ambegoda AMLC ${ }^{1}$, Kumara MSGR ${ }^{1}$, Sosai CP$^{2}$, \\ Abeygunasekera AM $\mathbf{M}^{1}$ \\ ${ }^{I}$ Department of Urology, ${ }^{2}$ Department of Pathology and ${ }^{3}$ Department of Radiology, Colombo South Teaching \\ Hospital, Dehiwala, Sri Lanka.
}

\author{
Correspondence: Dr.Anuruddha Abeygunasekera \\ e-mail: amabey@sltnet.lk \\ (1) https://orcid.org/0000-0003-3427-6796
}

\section{Introduction}

A colovesical fistula (CVF) is an abnormal connection between the colon and urinary bladder. Although they are uncommon, CVFs can cause significant morbidity, affect quality of life, and may lead to death, usually secondary to urosepsis $(1,2)$. CVF is the most common form of the enterovesical fistulae and occurs mostly due to diverticular disease $(65-80 \%)$, cancer $(10-20 \%)$ or Crohn's colitis (7 - 10\%) (2). Surgery, colonic stents, typhoid fever, syphilis and tuberculosis are rare causes of CVF. Although a CVF can be diagnosed clinically, imaging and endoscopy are often required to delineate the extent of a fistula and to elucidate its aetiology (3). Surgery is usually required to repair a CVF. We report a case of sigmoido-vesical fistula as a consequence of sigmoid tuberculosis.

\section{Case presentation}

A 54-year-old man developed frothy urine and passage of yellowish particles in his urine for one month. He did not have fever or other urinary symptoms such as dysuria, frequency, loin pain, lower abdominal pain or haematuria. There was no history of previous urinary tract infection or abdominal sepsis. There was no history of leg swelling or facial puffiness. His bowel habits were regular with no clinical evidence suggestive of colorectal malignancy, diverticulitis or tuberculosis. His general and system examination was normal. The abdominopelvic computed tomography with bladder contrast showed air in the bladder with no visible fistulous tract. Flexible sigmoidoscopy did not show any abnormality. Cystoscopy showed multiple fistulous openings in the dome and posterior wall of the bladder surrounded by areas of inflammatory changes. Preoperative chest X-ray was normal.
At laparotomy a colo-vesical fistula involving the sigmoid colon was evident. The fistulous tract and inflammatory mass was excised. Bladder defect was repaired and end to end colonic anastomosis was done. As the anastomotic site was low down in the pelvis a defunctioning ileostomy was done. Post-surgical period was uneventful. He went home on postoperative day three with the aim of keeping the urinary catheter for three weeks.

Few days later he developed fever. His abdominal and other system examinations were normal. His urine, blood and sputum cultures were negative. Ultra sound scan of the abdomen and pelvis did not show any intra-abdominal collections or any abnormality. The full blood count revealed white blood cells of $9.3 \times 10^{9} / \mathrm{L}$ (Normal range [NR] 4.0 $11.0 \times 10^{9} / \mathrm{L}$ ) with neutrophils $59 \%$; haemoglobin $10.6 \mathrm{~g} / \mathrm{dL}(\mathrm{NR} 13.5-16.5 \mathrm{~g} / \mathrm{dL})$ and platelet count of $436 \times 10^{9} / \mathrm{L}\left(\mathrm{NR} 150-450 \times 10^{9} / \mathrm{L}\right)$. C-reactive protein was $63 \mathrm{mg} / \mathrm{L}(\mathrm{NR}<5 \mathrm{mg} / \mathrm{L})$. ESR was 66 $\mathrm{mm} / 1^{\text {st }} \mathrm{hr}$. His Chest X-ray showed right side upper lobe consolidation. He was treated with intravenous antibiotics with poor response.

When the specimen was sectioned, a fistulous tract was identified, measuring $20 \mathrm{~mm}$ in length (Figure 1). The sections from the colovesical inflammatory mass showed large bowel mucosa with preserved crypt architecture. There was oedema of the lamina propria with dense acute on chronic inflammation (Figure 2). There was cryptitis and crypt abscesses too. Amidst this inflammation there were epithelioid cell granulomata with central caseating necrosis and Langhan type multinucleated giant cells (Figure 3 and 4). The inflammation extended through the mural wall. The margins of the resected colon were free of inflammation. Nine lymph nodes were found and they showed granuloma formation (Figure 5). 
However, Ziehl-Neelsen stain did not show acid fast bacilli. His Mantoux test showed an area of $10 \mathrm{~mm}$ induration. Three sputum and serial urine samples for acid fast bacilli were negative. After considering the clinical, radiological and histopathological findings, the patient was started on standard four drug anti-tuberculous therapy. His fever settled, weight increased and other symptoms subsided. He underwent ileostomy reversal after 6 weeks.

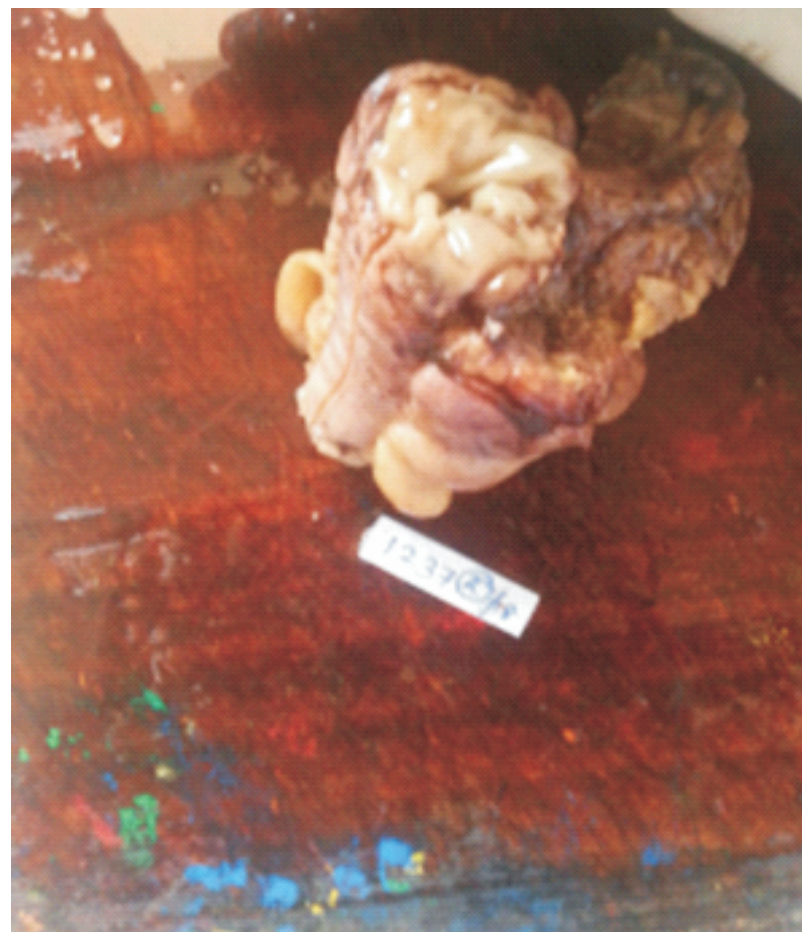

Figure 1: Macroscopic appearance of the resected specimen
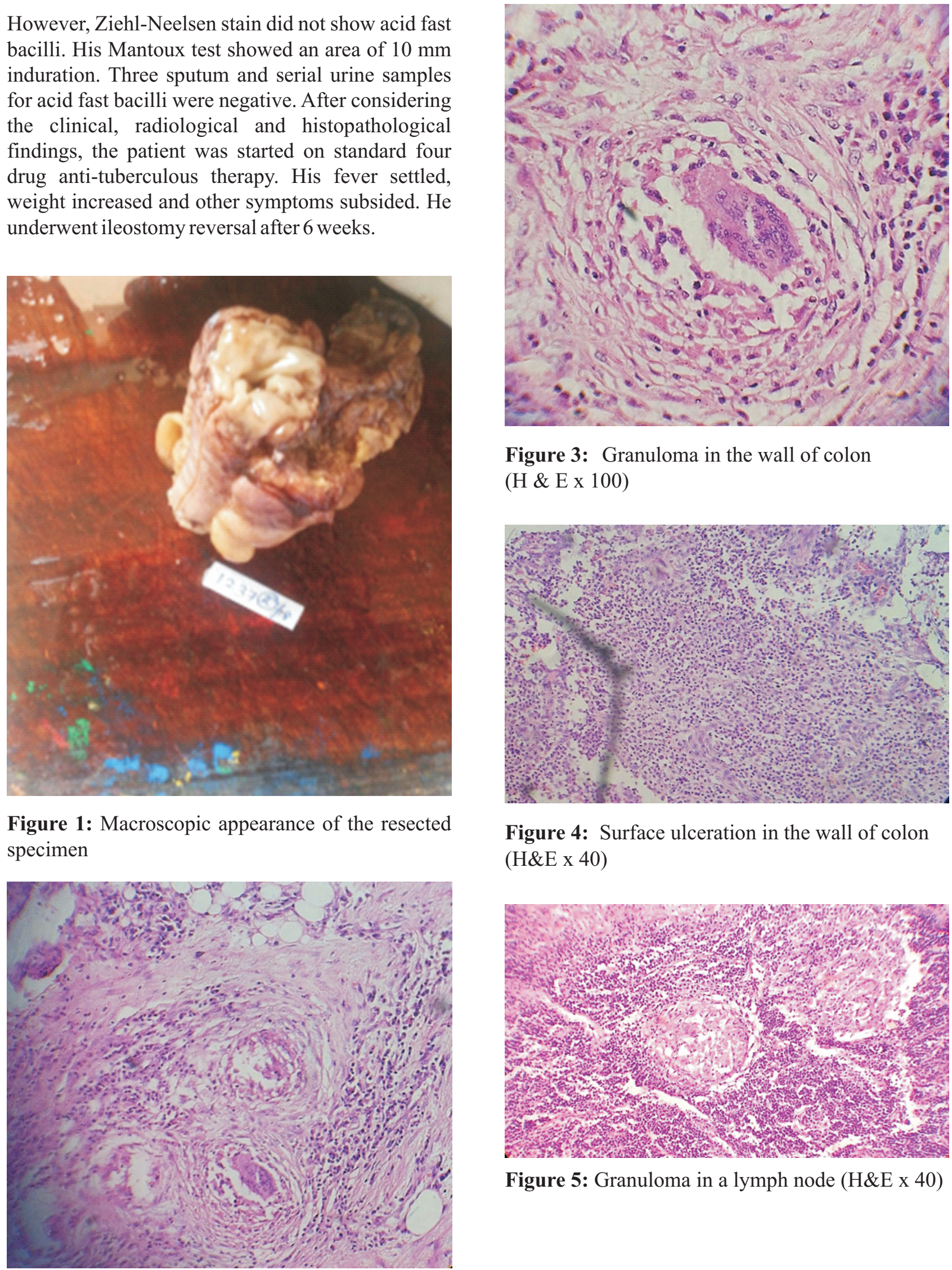

Figure 3: Granuloma in the wall of colon (H \& E x 100)

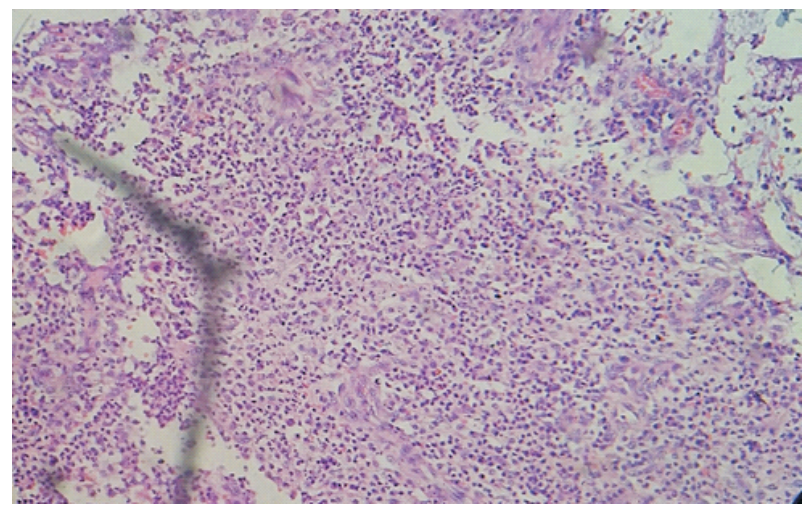

Figure 4: Surface ulceration in the wall of colon (H\&E x 40)

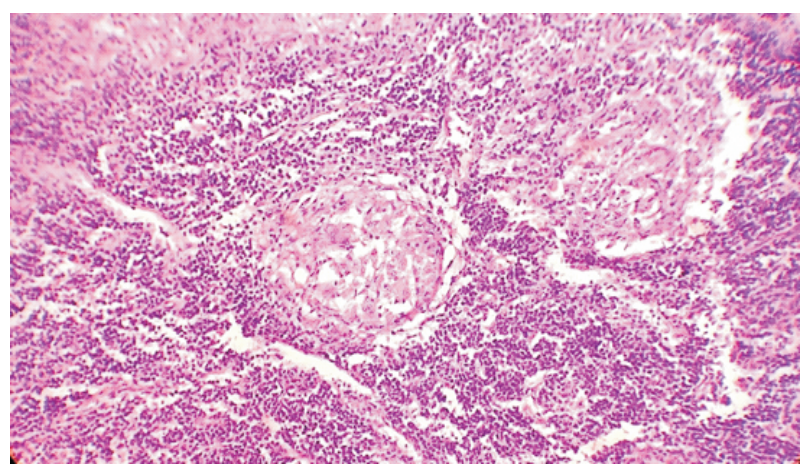

Figure 5: Granuloma in a lymph node (H\&E x 40)

Figure 2: Granuloma in the wall of colon (H\&Ex 40) 


\section{Discussion}

Segmental or isolated colonic tuberculosis refers to involvement of the colon without ileocaecal region, and constitutes $9.2 \%$ of all cases of abdominal tuberculosis $(4,5)$. It commonly involves the sigmoid, ascending and transverse colon. Multifocal involvement is seen in one third (28 to $44 \%$ ) of colonic tuberculosis. The median duration of symptoms is less than one year. Pain is the predominant symptom (78-90\%) and hematochezia occurs in less than one third $(4,5)$. Bleeding is frequently minor.

Colovesical is the most common form of the vesicoenteric fistulae and can result from diverticular disease (70-90\%), colorectal carcinoma $(20 \%)$ or Crohn's disease $(10 \%)(6)$. It is more common in men, with a ratio of $3: 1$ as uterus and broad ligaments act as a barrier between the sigmoid colon and the bladder. It is most common during the sixth and seventh decades of life and mean age of presentation is 55 to 75 years. Infections like typhoid fever, amoebiasis, syphilis, tuberculosis are rare causes of enterovesical fistulae (5-7). Biopsy helps in differentiating tuberculosis from Crohn's disease, and also excludes malignancy.

Patients with a CVF usually present with pneumaturia (50\% to $90 \%)$ and faecaluria $(40 \%$ $70 \%$ ). Other presentations are suprapubic pain, dysuria, urgency, frequency and haematuria (2). They may complain of gastrointestinal symptoms related to the underlying causes such as diverticulitis, colonic cancer, Chron's disease or tuberculosis. Diagnosis is confirmed by abdominopelvic computed tomography with oral/rectal or bladder contrast and it helps to diagnose around $72 \%$ patients with a CVF (2). Air in the bladder like in our patient may be a useful indicator when the fistulous tract is not obvious though its specificity is low. The lower gastrointestinal endoscopy has a low sensitivity in detecting a possible aetiology. However it is mandatory before planning surgical intervention in order to exclude a colonic malignancy eroding into the bladder. Cystoscopy is useful in detecting a fistula in $40 \%-80 \%$ of cases (3).

Treatment of CVF is primarily surgical after controlling the abdominal or urological sepsis with fluid resuscitation, antibiotics and drainage of bladder with a catheter. The specific operative procedure depends whether the fistula is benign or malignant. If it is a non-malignant cause, single stage bowel resection with primary anastamosis without diversion is a better option. If potential for leakage is high or if patient cannot tolerate a prolonged surgery, multi-stage procedures can be safer. Minimal access surgery depends on experienced hands but have 30\% conversion rate. Malignant fistulae are mostly due to colonic cancer and surgery should be based on usual oncological resection principles. Conservative treatment and endoscopic covered stenting of bowel for CVF is possible in selected patients but complications are more than surgical treatment. CVF of tuberculous origin is rare and our patient responded well to anti-tubercular treatment $(5,7)$.

This case report highlights the importance of considering tuberculosis as a cause of colovesical fistula in developing countries like ours, as well as the efficacy of medical therapy for controlling such an advanced stage of the disease. Similar to other sites of tuberculosis, patients with CVF also will not have all tests positive for tuberculosis. Hence the need to look at the full picture of the case is essential for proper management.

\section{Conclusions}

Extra pulmonary tuberculosis presenting as sigmoidovesical fistula is a rare clinical presentation despite the fact that tuberculosis is the most prevalent infectious disease in Sri Lanka. Due to its rarity and lack of specific features to prompt preoperative diagnosis, early evaluation of histopathology after surgery would prevent any delay in commencing antituberculous therapy.

\section{References}

1. Garcea G, Majid I, Sutton CD. Diagnosis and management of colovesical fistulae; six-year experience of 90 consecutive cases. Colorectal Dis, 2006; 8: 347.

2. Golabek T, Szymanska A, Szopinski T, Bukowczan J, Furmanek M, Pocoroznik J, Chlosta P. Enterovesical fistulae: aetiology, imaging, and management. Gastroenterol Res Pract, 2013; doi: 10.1155/293/ 617967. 
3. Melchior S, Cudovic D, Jones J, et al. Diagnosis and surgical management of colovesical fistulas due to sigmoid diverticulitis. Journal of Urology 2009; 182: 978.

4. Sharma MP, Bhatia V. Abdominal tuberculosis. Indian JMed Res, 2004; 120: 305-15.

5. Bandyopadhyay Sanjay, Das Chanchal, Maity Pranab K, Dhua Debargha, Sarkar Niladri. Isolated Colonic Tuberculosis with Colovesical Fistula. J Assoc Physicians India, 2010; 58: 396.
6. Karamchandani MC, West CF, Vesicoenteric fistula. Am Jour Surgery, 1984; 147: 681.

7. Balachandra Deepak, Nag Hulas Hirdaya, Sakhuja Puja, Barman Sandip. Tuberculosis of Intestine with Concurrent Complex Enterovesical and Enterocutaneous Fistula. Journal Clinical Diagnostic Res, 2018; 12: PD03-PD05. 